\title{
Spatio-temporal Alignment of Pedobarographic Image Sequences
}

Francisco P. M. Oliveira*, Andreia Sousa\#, Rubim Santos", João Manuel R. S. Tavares ${ }^{*}$

*Faculdade de Engenharia da Universidade do Porto (FEUP) / Instituto de Engenharia Mecânica e Gestão Industrial (INEGI), Rua Dr. Roberto Frias, 4200-465 Porto,

Portugal

${ }^{\#}$ Escola Superior da Tecnologia de Saúde do Porto (ESTSP), Instituto Politécnico do Porto (IPP), Centro de Estudos de Movimento e Actividade Humana (CEMAH), Rua Valente Perfeito, 322 - 4400-330 Vila Nova de Gaia, Portugal

Corresponding Author:

Professor João Manuel R. S. Tavares

Phone: +351225081487

Fax: +351225081445

Email: tavares@fe.up.pt

url: www.fe.up.pt/ tavares

The total number of words of the manuscript, including entire text from title page to figure legends: 4300 The number of words of the abstract: 237

The number of figures: 4

The number of tables: 1 


\begin{abstract}
This paper presents a methodology to align plantar pressure image sequences simultaneously in time and space. The spatial position and orientation of a foot in a sequence are changed to match the foot represented in a second sequence. Simultaneously with the spatial alignment, the temporal scale of the first sequence is transformed with the aim of synchronizing the two input footsteps. Consequently, the spatial correspondence of the foot regions along the sequences as well as the temporal synchronizing is automatically attained, making the study easier and more straightforward. In terms of spatial alignment, the methodology can use one of four possible geometric transformation models: rigid, similarity, affine or projective. In the temporal alignment, a polynomial transformation up to the $4^{\text {th }}$ degree can be adopted in order to model linear and curved time behaviors. Suitable geometric and temporal transformations are found by minimizing the mean squared error (MSE) between the input sequences. The methodology was tested on a set of real image sequences acquired from a common pedobarographic device. When used in experimental cases generated by applying geometric and temporal control transformations, the methodology revealed high accuracy. Additionally, the intra-subject alignment tests from real plantar pressure image sequences showed that the curved temporal models produced better MSE results $(p<0.001)$ than the linear temporal model. This paper represents an important step forward in the alignment of pedobarographic image data, since previous methods can only be applied on static images.
\end{abstract}

Keywords Biomechanics; Geometric and temporal transformations; Image registration; Intra-subject alignment; Plantar pressure. 


\section{Introduction}

The foot and ankle provide the necessary support and flexibility for weight-bearing and weight-shifting. Plantar pressure measurements provide relevant information on the foot and ankle role during gait and other functional activities [4, 22]. Although plantar pressure data is an important element in the assessment and prevention of ulceration of patients with diabetes $[1,5]$ and peripheral neuropathy, the information derived can also assist in the diagnosis and rehabilitation of impairments associated with various musculoskeletal, integumentary, and neurological disorders. The information gathered can be used to define suitable rehabilitation programs through alterations of footwear [1, 2], foot orthoses, exercise programs, and restrictions in the amount of weight-bearing $[19,20]$. Additionally, from a research perspective, the information is also useful to address questions regarding the relationship between plantar pressure and lowerextremity posture [14].

Usually, pedobarographic data can be converted to a discrete rectangular array at a point in time or over a period of time, giving rising to static images or to image sequences. In addition, efficient and robust techniques of image processing and analysis can assist clinicians and researchers to extract relevant information from images. For instance, methods of image alignment, i.e. methods to optimally align or register homologous image entities, can help in identifying the main plantar pressure areas and foot type. Furthermore, image alignment may assist clinicians in making accurate comparisons of a patient's plantar pressure distribution over time or between patients.

There are some studies on the alignment of pedobarographic image pairs; for example, those based on: principal axes transformation [6]; modal matching [3, 17, 23, 24]; principal axes combined with a search based on the steepest descent gradient optimization algorithm [15]; optimization based on genetic algorithms [16]; foot size and foot progression angle [8]; matching the contours represented in the input images [13]; optimization of the cross-correlation or phase correlation computed in the frequency domain [11]; and using a hybrid approach that combines a feature based solution with an intensity based solution [12].

The aforementioned solutions can only be used to align static pedobarographic images. Notwithstanding the value of the static information attained, when the footstep is considered in a natural progression, supplementary and pertinent information can be 
obtained, which may assist clinicians and researchers to carry out accurate studies on complete footsteps of patients before and after rehabilitation programs as well as making comparisons against well documented cases. In addition, the number of trials required to obtain reliable representations of the plantar pressure pattern is an important factor in dynamic data acquisition [9]. According to Hughes et al. [7], three to five walking trials enhances the reliability of the pressure measurement. As such, the spatiotemporal alignment of several trials of a subject can build a mean model image sequence automatically, which is more reliable than a single image sequence trial.

Despite the relevance of a computational spatio-temporal alignment of dynamic pedobarographic image sequences, as far as we know, no efficient or accurate solution has been proposed. This paper tries to overcome this limitation by proposing an efficient, accurate and fast computational solution for the spatio-temporal alignment of dynamic pedobarographic image sequences.

\section{Methods}

At first glance, to carry out the temporal alignment of two plantar pressure image sequences, one may be led to think that the first and last footstep images, i.e. the first and last images representing the footstep plantar pressure, of one sequence, need to be linearly transformed in the first and last footstep images of the second sequence. However, this simple approach would discard the information in the intermediate images, i.e. the plantar pressure distribution over time. Thus, in the proposed methodology, the temporal alignment is based on the pressure distribution of all the images in the sequences.

To align the footsteps represented in two image sequences the need for a time shift is evident, since the footsteps do not necessarily start at the same point of time in the two sequences, i.e. in the images with the same index in the sequences. In addition, subjects cannot be expected to walk at constant speeds, thus a linear time scaling is also needed. Furthermore, as small variations in speed can occur during footsteps, non-linear temporal transformations are required as well. Thus, linear and curved temporal transformations modeled by polynomials up to $4^{\text {th }}$ degree were integrated in the methodology. 


\subsection{Methodology}

The developed methodology entails the following steps (Fig. 1):

I) Build a peak pressure image representing the whole foot from each input image sequence;

II) Compute the spatial transformation that aligns the two peak pressure images built;

III) Compute an initial temporal alignment based on the linear mapping of the first and last images of the two footsteps;

IV) Use an optimization algorithm to find the parameters of the spatial and temporal transformations that optimize a (dis)similarity measure computed from the two sequences, starting from the spatial and temporal transformations previously found; V) Finally, perform the alignment of the input sequences in time and space using the optimal spatial and temporal transformations found.

(Insert Fig. 1 about here)

\subsubsection{Peak pressure image}

Let $S$ be a sequence of $n$ plantar pressure images, where $S(x, y, i)$ represents the pixel intensity (i.e. the related pressure at the correspondent sensor) at the spatial position $(x, y)$ of an image with index $i$ in the sequence $S$. Hence, the peak pressure image is given by $P(x, y)=\max (\{S(x, y, i): i=0, \ldots, n-1\})$.

\subsubsection{Initial spatial transformation}

The algorithm described in Oliveira and Tavares [12] is used to align the two peak pressure images. This 2D alignment algorithm can be divided into two main steps: First, an initial alignment is obtained by maximizing the cross-correlation between the peak plantar pressure images [11]. Afterwards, a multidimensional optimization algorithm is used to optimize the adopted (dis)similarity measure. The inputs of the optimization algorithm are the parameters of the initial geometric transformation computed in the 
previous step, and the outputs are the new parameters of the geometric transformation that optimize the (dis)similarity measure.

\subsubsection{Initial temporal shift and scaling}

The initial temporal transformation establishes a linear correspondence between the indexes of the images in the sequences to be aligned, and is found by considering that the first and last images of a footstep image sequence correspond to the first and last images of the second footstep image sequence, respectively. However, it should be noted that these first and last images of a footstep are not necessarily the initial and final images of the correspondent image sequence: Since, as we are only interested in images conveying relevant plantar information, found by evaluating their pixel intensity, the remainder images, e.g. the ones acquired before or after the interaction foot/ sensor plate, are discarded from the alignment process.

Therefore, by considering the temporal transformation $f$ and the first, $t_{1}$ and $s_{1}$, and the last, $t_{m}$ and $s_{n}$, images of the footsteps to be aligned, we have $f\left(s_{1}\right)=t_{1}$ and $f\left(s_{n}\right)=t_{m}$. Consequently, the transformation that represents a shift and a linear time scaling is given by a $1^{\text {st }}$ degree polynomial as:

$$
f(i)=\frac{t_{m}-t_{1}}{s_{n}-s_{1}} i+t_{1}-\frac{t_{m}-t_{1}}{s_{n}-s_{1}} s_{1}
$$

\subsubsection{Final optimization}

The spatial and temporal transformations obtained in the previous steps are then used as the initial solution in a multidimensional optimization algorithm. Hence, from this solution the optimization algorithm searches simultaneously and concurrently for the parameters of the spatial and temporal transformations that optimize the desired (dis)similarity measure. The optimization algorithm used is based on Powell's method, and the line optimization is carried out following Brent's method [18].

The spatial transformation model used to align the two input sequences can be rigid, similarity, affine or projective, and the time transformation can be modeled by polynomials up to the $4^{\text {th }}$ degree. The spatial transformation can be given in homogenous coordinates as: 


$$
\left[\begin{array}{l}
x^{\prime} \\
y^{\prime} \\
w
\end{array}\right]=\left[\begin{array}{lll}
a & b & e \\
c & d & f \\
r & q & 1
\end{array}\right]\left[\begin{array}{l}
x \\
y \\
1
\end{array}\right],
$$

where $\left[\begin{array}{lll}x & y & 1\end{array}\right]^{T}$ and $\left[\begin{array}{lll}x^{\prime} & y^{\prime} & w\end{array}\right]^{T}$ are the original and the transformed homogeneous coordinates. In this equation, $a, b, c$ and $d$ are parameters that represent the deformation, $e$ and $f$ stand for the spatial shift, and $r$ and $q$ define the projection point. For rigid, similarity and affine transformations, the parameters $r$ and $q$ are set equal to 0 (zero). The polynomial model adopted for the temporal transformation is given by:

$$
i^{\prime}=f(i)=a_{4} i^{4}+a_{3} i^{3}+a_{2} i^{2}+a_{1} i+a_{0},
$$

where $i$ and $i^{\prime}$ are the image indexes in the original and transformed sequences, and $a_{4}, a_{3}, a_{2}, a_{1}$ and $a_{0}$ are the coefficients of the $4^{\text {th }}$ degree polynomial. For lower degree polynomials, the higher degree coefficients are set as constants with a value equal to 0 (zero).

Two different schemes were set up to optimize the temporal alignment: an unconstrained and a constrained optimization scheme. In the former, all parameters of the adopted polynomial model can vary independently. In the latter, the first and last images of a footstep must map the first and last images, respectively, of the second footstep.

It should be noted that using the constrained optimization scheme, if a $1^{\text {st }}$ degree polynomial is chosen as the temporal transformation model, then only one solution exists (Equation 1) and the spatial optimization is performed solo.

\subsection{Dissimilarity measure}

In the results presented in this work, the MSE among the pixel intensity values was used as the dissimilarity measure; however, another intensity based measure could be considered. Let $T$ and $S$ be two discrete image sequences of $N \times M \times Z$ pixels. The adopted MSE is given as:

$$
M S E=\frac{1}{N \times M \times Z} \sum_{x}^{N} \sum_{y}^{M} \sum_{i}^{Z}(T(x, y, i)-S(x, y, i))^{2} .
$$

Thus, the lower the MSE value is, the better aligned the input image sequences are. 


\subsection{Dataset}

The experimental dataset was acquired using an EMED system (Novel GmbH, Germany) with a spatial resolution of 2 sensors per $\mathrm{cm}^{2}$, and a pressure sensibility of 5 $\mathrm{kPa}$ with minimum threshold value of $10 \mathrm{kPa}$. The pressure measurement technology of this system offers good reliability for most force/pressure variables when a single measurement is used, and an excellent reliability when the mean value of three or more measurements is used [7].

The dataset of 168 image sequences was acquired at frequency rate of 25 frames per second from 28 subjects with three image sequences representing each foot of each subject at normal walking speed. The sample included 7 men (18.4 \pm 0.5 years, $173 \pm 7$ $\mathrm{cm}, 68.6 \pm 6.0 \mathrm{~kg})$ and 21 women $(20.4 \pm 2.3$ years, $164 \pm 5 \mathrm{~cm}, 58.3 \pm 6.3 \mathrm{~kg})$, who were selected according to: no history of recent osteoarticular or musculotendon injury of the lower limb or signs of neurological dysfunction which could affect lower limb motor performance; no history of lower limb surgery, of lower limb anatomical deformities, congenital or acquired, or any other disability that might in some way affect gait; absence of callus formation on plantar pressure surface [27].

Before the data acquisition, all subjects walked over the pedobarographic system several times until they felt comfortable under the experiment conditions. The subjects were invited to walk at a normal pace along a walkway and were asked to look straight ahead while walking. Each subject performed two series of three trials. The order of the series was randomized, and it was guaranteed that only one foot had contact on the pressure system at a time. Normal speed was selected as a number of authors have shown that plantar pressure distribution is dependent upon walking speed [10, 21, 25].

The study was conducted according to the ethical norms of the Institutions involved and the Declaration of Helsinki, and informed consent was obtained from all participants.

\subsection{Alignment accuracy assessment using control image sequences}

The alignment accuracy was assessed by applying a set of spatial and temporal control transformations to a real pedobarographic image sequence randomly chosen from the dataset. Afterwards, the transformed sequences were aligned with the original sequence. Then, the spatial and temporal transformations obtained were compared against the control transformations. The residual error (RE), that is, the square root of the mean 
squared difference between the expected position for each pixel and the estimated position from the proposed solution, was used to assess the accuracy.

The temporal control transformations were chosen to simulate the natural speed variations that can occur on footsteps during normal walking, and the spatial control transformations were defined to simulate the walking along any direction. Additionally, to simulate the real behavior of pedobarographic systems, the transformed image sequences were pre-processed before the alignment process: pixel intensities were rounded off to multiples of $5 \mathrm{kPa}$ (representing the addition of noise uniformly distributed between -2.5 and $2.5 \mathrm{kPa}$ ) and the intensities inferior to $10 \mathrm{kPa}$ were set equal to 0 (zero).

\subsection{Alignment quality assessment using real image sequences}

In the tests regarding the quality assessment, just pairs of sequences of the same subject were aligned; that is, intra-subject image sequences alignment. Hence, the goal was to search for the geometric and temporal transformations that generate the best results, i.e. the minimum MSE value. In all alignment experiments, a rigid model was considered for the spatial alignment, since intra-subject alignment was to be performed. The accuracy was statistically compared using two-sided $t$ tests. Additionally, the alignment quality was also accessed by visual evaluation.

Six different alignment experiments were done per subject, three per foot. Thus, there were 168 image sequence pairs in total for the intra-subject alignment experiments.

\subsection{Implementation}

The methodology developed was fully implemented in $\mathrm{C}^{++}$, using Microsoft Visual Studio 8, and tested on a PC notebook with an AMD Turion $642.0 \mathrm{GHz}$ microprocessor, 1.0 GB of RAM and running Microsoft Windows XP. In the experiments described in the next section, the image transformations were performed using bilinear interpolation resampling [26]. 


\section{Results}

\subsection{Accuracy assessment using control image sequences}

Table 1 shows the maximum RE obtained for all tests done using the spatial and temporal control transformations. The time scale of the control sequence was warped using four models: $f_{1}(i)=1.15 i-0.5, f_{2}(i)=-0.04 i^{2}+2 i-1$,

$f_{3}(i)=-0.0025 i^{3}+0.1 i^{2}+0.125 i+0.5$ and $f_{4}(i)=i+3 \sin \left(\frac{i}{5}\right)$, where $i$ is the image index in the original sequence (Fig. 2), and, for each, ten rotation angles were used to warp the space domain: $5^{\circ}, 41^{\circ}, 77^{\circ}, 113^{\circ}, \ldots, 329^{\circ}$. This way, 40 warped control sequences were built.

The temporal warp control transformations used were chosen in accordance to the expected walking speed variations. As can be seen in Figure 2, the functions used traduce the usual speed variations along footstep sequences; for instance, relatively to the original footstep sequence, $f_{4}(i)$ decreases the speed at the beginning of the footstep and increases the speed at the end.

In the first experiment, the 10 image sequences warped by the selected rotation angles and the temporal transformation $f_{1}(i)$ were used. Then the developed alignment framework was successively configured to use each of the adopted temporal alignment models and optimization schemes. The higher RE values for each temporal model/optimization scheme combination were stored. The following three experiments done were similar to this one, but using the sequences temporally warped by the functions $f_{2}(i), f_{3}(i)$ and $f_{4}(i)$, instead (Table 1$)$.

(Insert Fig. 2 and Table 1 about here)

\subsection{Alignment quality assessment using real image sequences}

There are no reference values to evaluate the accuracy of the geometric and temporal transformations obtained from the alignment of real pedobarographic image sequences. 
Therefore, the alignment accuracy of the methodology was assessed from the MSE values (Fig. 3).

(Insert Fig. 3 about here)

The intra-subject alignment tests were carried out using a rigid transformation model for the spatial alignment and all four polynomial temporal models with the constrained and unconstrained optimization schemes were used (Fig. 3). Figure 4 shows an example of the alignment obtained from two pedobarographic image sequences. From this figure, one can realize that the sequence aligned using a $4^{\text {th }}$ degree temporal transformation model with unconstrained optimization is visually more similar to the reference sequence than the sequence aligned using a $1^{\text {st }}$ degree temporal transformation model with constrained optimization.

(Insert Fig. 4 about here)

The average computational processing times for the intra-subject alignment with the unconstrained optimization scheme were: $2.1 \pm 0.6,4.4 \pm 1.3,8.1 \pm 2.5$ and $11.2 \pm 4.7$ seconds, using $1^{\text {st }}, 2^{\text {nd }}, 3^{\text {rd }}$ and $4^{\text {th }}$ degree polynomials, respectively. Using the constrained optimization scheme instead, the processing times were: $0.9 \pm 0.3,1.1 \pm 0.3$, $2.7 \pm 0.9$ and $5.7 \pm 1.6$ seconds, respectively.

\section{Discussion}

The methodology proposed revealed to be very accurate in the spatio-temporal alignment of pedobarographic image sequences, mainly when the unconstrained optimization scheme is used, as is confirmed in Table 1.

In the tests using the spatial and temporal control warp transformations and the polynomials of the $3^{\text {rd }}$ and $4^{\text {th }}$ degrees as temporal models, the maximum spatial RE values were equal to 0.0061 pixel (approximately $0.043 \mathrm{~mm}$ ) and 0.021 pixel (around $0.148 \mathrm{~mm}$ ) considering the unconstrained and constrained optimization schemes, respectively (Table 1). The maximum temporal RE value was also very low when 
polynomials of $3^{\text {rd }}$ and $4^{\text {th }}$ degree were considered as temporal models together with the unconstrained optimization scheme (Table 1).

The temporal RE values obtained on using the unconstrained optimization scheme were always inferior to the values obtained when the constrained optimization scheme was used. This was already expected, since the temporal scale is discrete ( $25 \mathrm{fps}$ ) and so, the first and last images of a footstep can be associated to any point of time in a period of $40 \mathrm{~ms}$.

The visual evaluation of the resultant intra-subject alignments from the real image sequences showed that the curved temporal transformations are more suitable than the linear temporal transformation. In fact, in most cases, the visual similarity between the aligned sequences was superior when curved temporal models were used instead of the linear temporal model. In the remaining cases, the visual similarity between the aligned sequences was indistinguishable.

By assessing the accuracy of the alignment results from real image sequences based on the MSE, we concluded that higher degree polynomials produced lower MSE values ( $p<0.001$ ), independently of the optimization scheme used (Fig. 3). From the mean MSE values presented in Figure 3, one can see that for each type of temporal transformation model adopted, the MSE values obtained using the unconstrained optimization scheme were lower $(p<0.001)$ than the correspondent values obtained using the constrained optimization scheme. This is in agreement with the results obtained using the control transformations, which proves the superior accuracy of the unconstrained optimization scheme. Additionally, from Figure 3 one can realize that the mean MSE undertaken a small reduction with the increasing of the polynomial degree; however, an exhaustive analysis on the experimental results revealed that in the alignment of some footsteps that reduction was significant.

Although this methodology permits the use of similarity, affine and projective transformations, we did not use them in the experiments, since the goal was the alignment of pedobarographic image sequences from the same foot. Nevertheless, these non-rigid spatial transformations are useful in the alignment of image sequences from different feet.

In this work, the MSE was used as the image dissimilarity measure to be minimized since in previous works it was shown to be very suitable for the alignment of plantar 
pressure images $[12,16]$. Besides, the squared root of the MSE represents the mean pressure differences between the plantar pressure images that are relevant biomechanical information and important for statistical analysis. However, as already mentioned, other intensity based measures could be considered.

Even using a not up-to-dated PC, the processing time was always quite low. Thus, the low processing time and the high accuracy guarantee that the proposed spatio-temporal alignment methodology is appropriate for pedobarographic image sequence studies in clinics or laboratories.

\section{Acknowledgements}

This work was partially done under the scope of the following research projects: "Methodologies to Analyze Organs from Complex Medical Images - Applications to the Female Pelvic Cavity", "Cardiovascular Imaging Modeling and Simulation - SIMCARD" and "Aberrant Crypt Foci and Human Colorectal Polyps: Mathematical Modelling and Endoscopic Image Processing”, with references PTDC/EEA-CRO/103320/2008, UTAustin/CA/0047/2008 and UTAustin/MAT/0009/2008, respectively, financially supported by Fundação para a Ciência e a Tecnologia in Portugal.

The first author would like to thank Fundação Calouste Gulbenkian in Portugal for his $\mathrm{PhD}$ grant.

\section{References}

1. Actis R, Ventura L, Lott D, Smith K, Commean P, Hastings M, Mueller M (2008) Multi-plug insole design to reduce peak plantar pressure on the diabetic foot during walking. Med Biol Eng Comput 46:363-371

2. Actis R, Ventura L, Smith K, Commean P, Lott D, Pilgram T, Mueller M (2006) Numerical simulation of the plantar pressure distribution in the diabetic foot during the push-off stance. Med Biol Eng Comput 44:653-663

3. Bastos L, Tavares J (2004) Improvement of modal matching image objects in dynamic pedobarography using optimization techniques. Lecture Notes in Computer Science 3179/2004. Springer, Berlin, pp 39-50

4. Duckworth T, Betts R, Franks C, Burke J (1982) The measurement of pressure under the foot. Foot Ankle 3(3):130-141

5. Duckworth T, Boulton A, Betts R, Franks C, Ward J (1985) Plantar pressure measurements and the prevention of ulceration in the diabetic foot. The J Bone Jt Surg 67-B(1):79-85

6. Harrison A, Hillard P (2000) A moment-based technique for the automatic spatial alignment of plantar pressure data. Proc Inst Mech Eng H: J Eng Med 214(3):257-264

7. Hughes J, Pratt L, Linge K, Clark P, Klenerman L (1991) Reliability of pressure measurements: the EMED F system. Clin Biomech 6(1):14-18

8. Keijsers N, Stolwijk N, Nienhuis B, Duysens J (2009) A new method to normalize plantar pressure measurements for foot size and foot progression angle. J Biomech 42(1):87-90

9. McPoil T, Cornwall M, Dupuis L, Cornwell M (1999) Variability of plantar pressure data. A comparison of the two-step and midgait methods. J Am Podiatric Med Assoc 89(10):495-501

10. Morag E, Cavanagh P (1999) Structural and functional predictors of regional peak pressures under the foot during walking. J Biomech 32(4):359-370 
11. Oliveira F, Pataky T, Tavares J (2010) Registration of pedobarographic image data in the frequency domain. Comput Methods Biomech Biomed Eng 13(6):731-740

12. Oliveira F, Tavares J (2011) Novel framework for registration of pedobarographic image data. Med Biol Eng Comput 49(3): 313-323

13. Oliveira F, Tavares J, Pataky T (2009) Rapid pedobarographic image registration based on contour curvature and optimization. J Biomech 42(15):2620-2623

14. Orlin M, McPoil T (2000) Plantar pressure assessment. Phys Therapy 80(4):399-409

15. Pataky T, Goulermas J (2008) Pedobarographic statistical parametric mapping (pSPM): a pixel-level approach to foot pressure image analysis. J Biomech 41(10):2136-2143

16. Pataky T, Goulermas J, Crompton R (2008) A comparison of seven methods of within-subjects rigidbody pedobarographic image registration. J Biomech 41(14):3085-3089

17. Pinho R, Tavares J (2004) Dynamic pedobarography transitional objects by Lagrange's equation with FEM, modal matching and optimization techniques. Lecture Notes in Computer Science 3212/2004. Springer, Berlin, pp 92-99

18. Press W, Teukolsky S, Vetterling W, Flannery B (1992) Numerical recipes in C: the art of scientific computing, 2nd ed. Cambridge University Press, New York

19. Putti A, Arnold G, Abboud R (2010) Foot pressure differences in men and women. Foot Ankle Surg $16(1): 21-24$

20. Rosenbaum D, Becker H (1997) Plantar pressure distribution measurements. Technical background and clinical applications. Foot Ankle Surg 3(1):1-14

21. Rosenbaum D, Hautmann S, Gold M, Claes L (1994) Effects of walking speed on plantar pressure patterns and hindfoot angular motion. Gait Posture 2(3):191-197

22. Soames R (1985) Foot pressure patterns during gait. J Biomed Eng 7(2):120-126

23. Tavares J, Barbosa J, Padilha A (2000) Matching image objects in dynamic pedobarography. in 11th Portuguese Conference on Pattern Recognition (RecPAD 2000), Porto, Portugal

24. Tavares J, Bastos L (2010) Improvement of modal matching image objects in dynamic pedobarography using optimization techniques. in: Bunke H, Villanueva J, Sánchez G, Otazu X (eds), Progress in Computer Vision and Image Analysis. World Scientific, Singapore, pp 339-368

25. Taylor A, Menz H, Keenan A (2004) The influence of walking speed on plantar pressure measurements using two-step gait initation protocol. The Foot 14(1):49-55

26. Thévenaz P, Blu T, Unser M (2000) Interpolation revisited. IEEE Trans Med Imaging 19(7):739-758

27. Young M, Cavanagh P, Thomas G, Johnson M, Murray H, Boulton A (1992) The effect of callus removal on dynamic plantar foot pressures in diabetic patients. Diabet Medicine 9(1):55-57 


\section{FIGURE CAPTIONS}

Fig. 1 Proposed methodology for the spatio-temporal alignment of pedobarographic image sequences

Fig. 2 Representation of the temporal warp functions used as control transformations in the temporal region of interest

Fig. 3 Mean MSE values obtained by using each temporal transformation model in the alignment of 168 pairs of real pedobarographic image sequences. (Only the pixels with non-zero value were used in the MSE calculus.)

Fig. 4 Two alignment examples from pedobarographic image sequences: In the first row, the sequence used as reference; in the second row, the sequence to be aligned; in the third row, the aligned sequence using a $1^{\text {st }}$ degree temporal transformation model with constrained optimization; and finally, in the last row, the aligned sequence using a $4^{\text {th }}$ degree temporal transformation model with unconstrained optimization. (To simplify the visualization, only half of all images are shown) 


\section{TABLE CAPTION}

Table 1 Maximum residual errors obtained in the alignment of image sequences that were synthetically spatio-temporal warped 
Table 1

\begin{tabular}{|c|c|c|c|c|c|}
\hline \multirow{2}{*}{$\begin{array}{l}\text { Applied temporal } \\
\text { transformation }\end{array}$} & \multirow{2}{*}{$\begin{array}{l}\text { Degree of the } \\
\text { polynomial } \\
\text { model used in } \\
\text { the temporal } \\
\text { alignment }\end{array}$} & \multicolumn{2}{|c|}{$\begin{array}{l}\text { Unconstrained } \\
\text { optimization }\end{array}$} & \multicolumn{2}{|c|}{ Constrained optimization } \\
\hline & & $\begin{array}{l}\text { Maximum } \\
\text { spatial RE } \\
\text { [pixel] }\end{array}$ & $\begin{array}{c}\text { Maximum } \\
\text { temporal RE } \\
{[\mathrm{s}]}\end{array}$ & $\begin{array}{c}\text { Maximum } \\
\text { spatial RE } \\
\text { [pixel] }\end{array}$ & $\begin{array}{c}\text { Maximum } \\
\text { temporal RE } \\
{[\mathrm{s}]}\end{array}$ \\
\hline \multirow{4}{*}{$f_{1}(i)$} & 1 & 0.0017 & 0.0002 & 0.0367 & 0.0112 \\
\hline & 2 & 0.0017 & 0.0002 & 0.0119 & 0.0083 \\
\hline & 3 & 0.0017 & 0.0003 & 0.0071 & 0.0052 \\
\hline & 4 & 0.0016 & 0.0003 & 0.0075 & 0.0049 \\
\hline \multirow{4}{*}{$f_{2}(i)$} & 1 & 0.0629 & 0.0501 & 0.9018 & 0.2211 \\
\hline & 2 & 0.0022 & 0.0002 & 0.0221 & 0.0124 \\
\hline & 3 & 0.0021 & 0.0003 & 0.0183 & 0.0104 \\
\hline & 4 & 0.0024 & 0.0020 & 0.0135 & 0.0073 \\
\hline \multirow{4}{*}{$f_{3}(i)$} & 1 & 0.0096 & 0.0127 & 0.1154 & 0.0435 \\
\hline & 2 & 0.0119 & 0.0080 & 0.0371 & 0.0200 \\
\hline & 3 & 0.0024 & 0.0002 & 0.0031 & 0.0025 \\
\hline & 4 & 0.0028 & 0.0014 & 0.0026 & 0.0019 \\
\hline \multirow{4}{*}{$f_{4}(i)$} & 1 & 0.0228 & 0.0540 & 0.1161 & 0.0860 \\
\hline & 2 & 0.0682 & 0.0340 & 0.0747 & 0.0485 \\
\hline & 3 & 0.0061 & 0.0056 & 0.0188 & 0.0104 \\
\hline & 4 & 0.0049 & 0.0030 & 0.0201 & 0.0095 \\
\hline
\end{tabular}




\section{FIGURES}

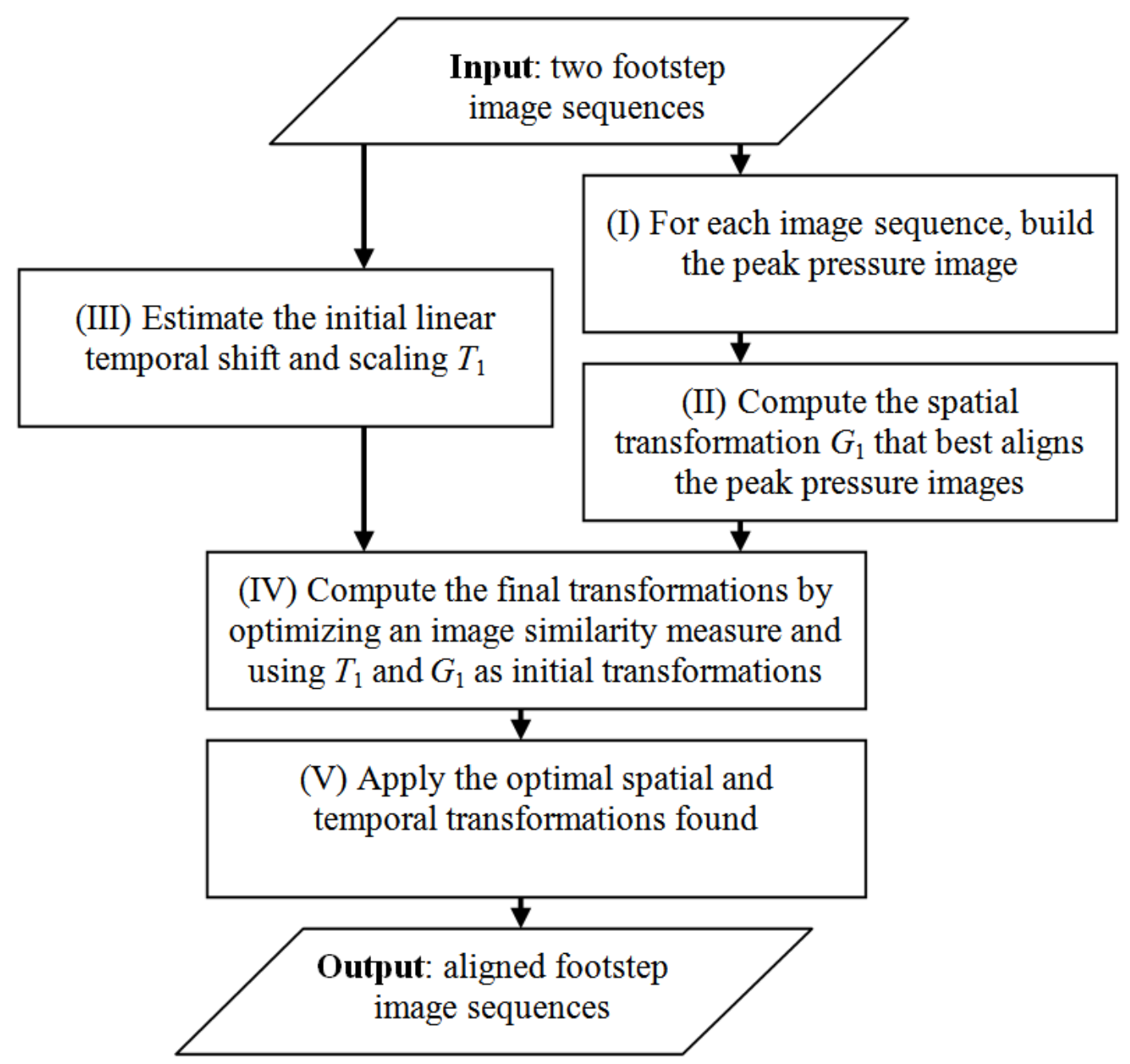

Figure 1 


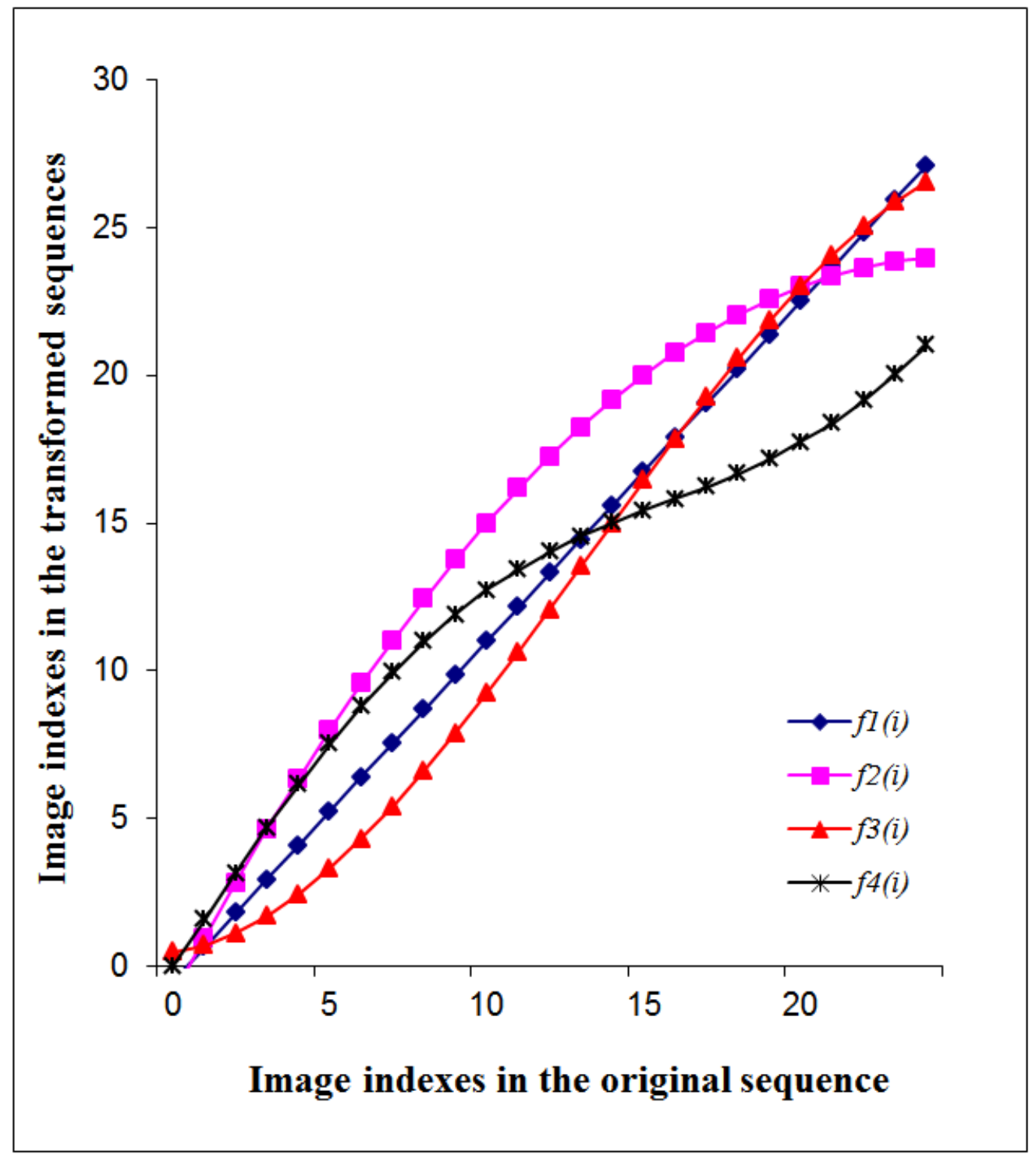

Figure 2 


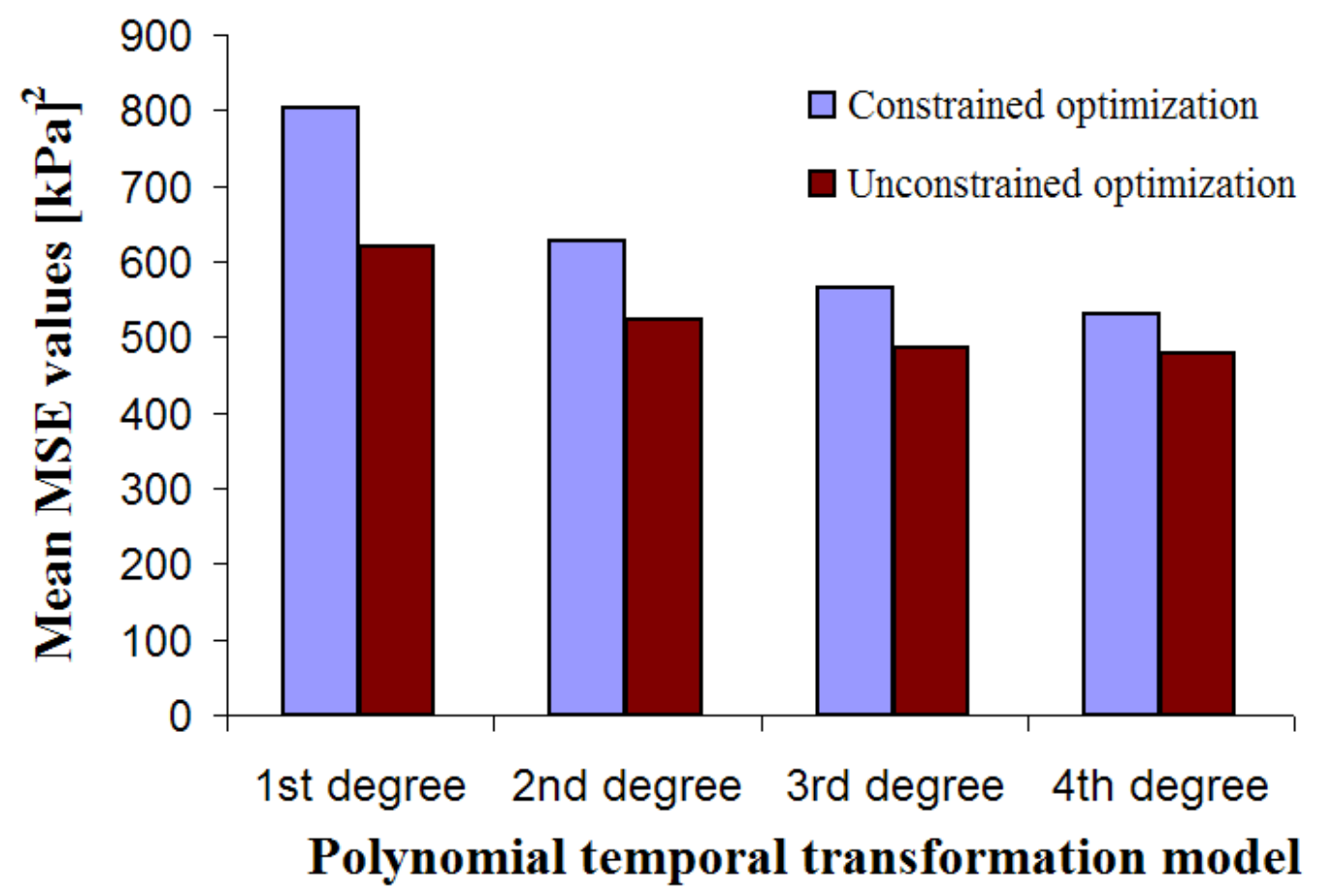

Figure 3

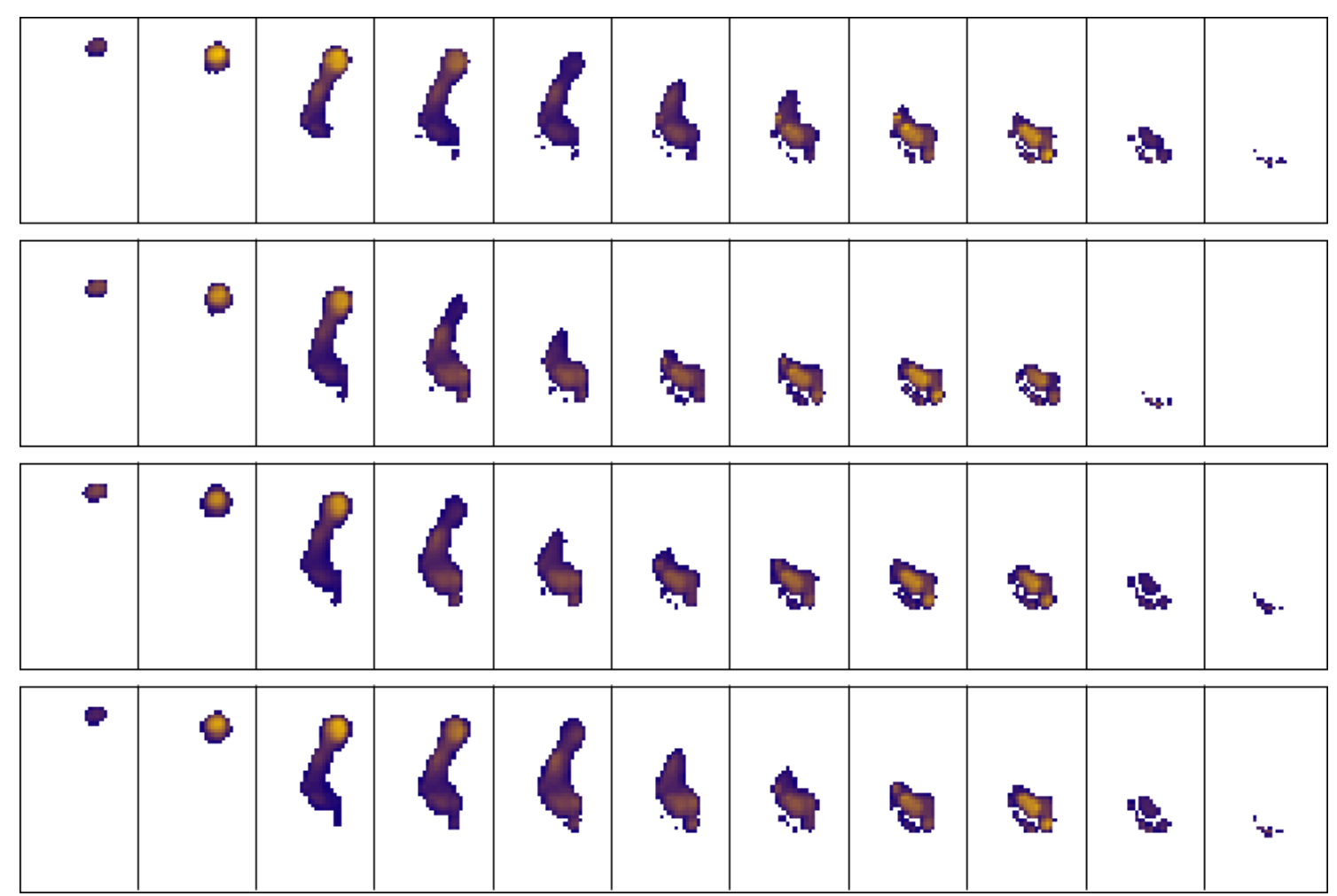

Figure 4 